\title{
Robust Global Nonlinear Sampled-Data Regulator for the Glucose-Insulin System
}

M. Di Ferdinando
P. Pepe

P. Palumbo
S. Panunzi

A. De Gaetano

\begin{abstract}
In this paper we deal with the problem of tracking a desired plasma glucose evolution by means of intra-venous insulin administration, for Type 2 diabetic patients exhibiting basal hyperglycemia. A nonlinear time-delay model is used to describe the glucose-insulin regulatory system, and a modelbased approach is exploited in order to design a global sampleddata control law for such system. Sontag's universal formula is designed to obtain a steepest descent feedback induced by a suitable control Lyapunov-Krasovskii functional. Such a feedback is a stabilizer in the sample-and-hold sense. Furthermore, the input-to-state stability redesign method is used in order to attenuate the effects of bounded actuation disturbances and observation errors, which can appear for uncertainties in the instruments. The proposed control law depends on sampled glucose and insulin measurements. Theoretical results are validated through simulations.
\end{abstract}

\section{INTRODUCTION}

Diabetes Mellitus (DM) is a chronic disease, whose alarming continuous growth has been estimated to involve currently 380 million patients worldwide [1]: as a matter of fact, diabetes management has a heavy impact on many national public health budgets. DM is in fact a group of metabolic disorders characterized by hyperglycemia, since it involves a complete lack of the hormone insulin or a (partial or severe) disruption of the endogenous glucose control feedback exerted by insulin secreted by the pancreas. The former case refers to Type 1 DM (T1DM) and requires an exogenous supply of insulin for T1DM patients to survive. The latter case refers to Type 2 DM (T2DM), and it is caused by a combination of resistance to insulin action and inadequate compensatory insulin secretory response: though less severe than T1DM, T2DM accounts for $85 \%$ to $95 \%$ of all cases of diabetes, and its societal impact is therefore huge. And a timely control of the hyperglycemia prevents or hinders the emergence of diabetic complications (retinopathy, neuropathy, nefropathy, ...) in both T1DM and T2DM. The Artificial Pancreas (AP) refers to the set of integrated systems combining the design of the insulin infusion therapy, the actuators in charge of its delivery (insulin pumps) and the sensor equipments providing measurements to the controller

This work is supported in part by the Center of Excellence for Research DEWS. Tel.: +39 0862434422;fax: +39 0862434403. E-mail address: mario.diferdinando@graduate.univaq.it

M. Di Ferdinando and Pierdomenico Pepe are with the Department of Information Engineering, Computer Science, and Mathematics, University of L'Aquila, L'Aquila, Italy (e-mail: pierdomenico.pepe@univaq.it, mario.diferdinando@graduate.univaq.it).

P. Palumbo, S. Panunzi and A. De Gaetano are with the Institute of Systems Analysis and Computer Science, National Research Council, Rome, Italy (e-mail: pasquale.palumbo@iasi.cnr.it, simona.panunzi@biomatematica.it, andrea.degaetano@biomatematica.it). in order to synthesize the closed-loop control law. Most of the available AP results actually involve T1DM (see, among the others, [6], [9], [12], [15], [38] and references therein). This work investigates closed-loop glucose control therapies for T2DM patients, by means of a model-based approach, that means the control law is synthesized by properly exploiting the mathematical model of the glucoseinsulin system. The chosen model is a Delay Differential Equation (DDE) model, published in [23], [26]: motivation is that DDE models are known to properly account for the endogenous insulin delivery rate [16], [21], which cannot be neglected for T2DM. Moreover, the DDE model here adopted has already been shown to be effective in designing model-based glucose control laws [22], [24] according to continuous glucose measurements and continuous insulin administration. Differently from [22], [24], here the proposed regulator is synthesized according to a different control design architecture, on the ground of sampled-data measurements (sampled-data regulator). Preliminary results have been proposed in [31] where a local sampled-data control law for the nonlinear DDE model is presented, whilst here the problem to consider a global sampled-data control law is investigated. To the best of our knowledge, this problem has not been addressed in the literature. Insulin is supposed to be intravenously administered: the intravenous route provides a wider range of possible strategies with respect to the subcutaneous route, and ensures a rapid delivery with negligible delays. As a matter of fact, control algorithms based on intravenous infusions (we can cite, among the others, [3], [10], [14], [22], [24], [27], [34]) are directly applicable so far only to problems of glycemia stabilization in critically ill subjects, such as in surgical Intensive Care Units after major procedures, [37]. Furthermore, in order to cope with technical hurdles, the following pair of uncertainties has been considered. From one hand, a disturbance is added to the insulin kinetics in order to model errors concerning both the endogenous insulin delivery rate and the mechanism actuating the insulin pump. Such a framework has been considered in [25], where an input-to-state stability redesign method is proposed, on a continuous-time basis. On the other hand, errors have been assumed to affect the measurements, usually provided by uncertainties in the instruments. The sampled-data regulator will account for both these sources of possible malfunctioning. Sampled-data stabilization has been studied in the literature by many approaches, such as: i) the time-varying delay approach (see for instance [11]), ii) the approximate system discretization approach (see [18], [19]), iii) the hybrid system approach (see [2], [20]); iv) the 
stabilization in the sample-and-hold sense approach (see [5], [8], [29]). The notion of stabilization in the sample-and-hold sense, introduced in 1997 in [5], has been widely studied for systems described by ordinary differential equations, and recently extended to systems with delays too (see [7], [8], [28] and [29]). In this paper, taking into account the results in [7], concerning the stabilization in the sample-and-hold sense for nonlinear time-delay systems, a robust nonlinear global state-feedback stabilizer for the DDE model of the glucoseinsulin system is provided. The proposed global sampleddata controller is based on the theory of Sontag's stabilizers (see [17], [30] and [35]) and on the recent results about stabilization in the sample-and-hold sense for nonlinear timedelay systems (see [7], [8], [28] and [29] and reference therein). The feedback control law shows good performances for sampling periods comparable to the ones available for usual glucose sensors, with the robustified sampled-data state feedback that can cope with the effect of significant actuation disturbances and measurement errors affecting both the glucose and insulin concentrations.

Notation $\mathbb{N}$ denotes the set of nonnegative integer numbers, $\mathbb{R}$ denotes the set of real numbers, $\mathbb{R}^{\star}$ denotes the extended real line $[-\infty,+\infty], \mathbb{R}^{+}$denotes the set of nonnegative reals $[0,+\infty)$. The symbol $|\cdot|$ stands for the Euclidean norm of a real vector, or the induced Euclidean norm of a matrix. For a positive integer $n$, for a positive real $\Delta$, a Lebesgue measurable function $f:[-\Delta, 0] \rightarrow R^{n}$ is said to be essentially bounded if ess $\sup _{t \in[-\Delta, 0]}|f(t)|<+\infty$, where ess $\sup _{t \in[-\Delta, 0]}|f(t)|=\inf \left\{a \in R^{*}: \lambda(\{t \in[-\Delta, 0]:\right.$ $|u(t)|>a\})=0\}, \lambda$ denoting the Lebesgue measure. The essential supremum norm of an essentially bounded function is indicated with the symbol $\|\cdot\|_{\infty}$. For a positive integer $n$, for a positive real $\Delta$ (maximum involved time-delay): $\mathcal{C}$ and $W^{1, \infty}$ denote the space of the continuous functions mapping $[-\Delta, 0]$ into $\mathbb{R}^{n}$ and the space of the absolutely continuous functions, with essentially bounded derivative, mapping $[-\Delta, 0]$ into $\mathbb{R}^{n}$, respectively; $\mathcal{Q}$ denotes the space of bounded, right-continuous functions, with possibly a finite number of points with jump-type discontinuity, mapping $[-\Delta, 0)$ into $\mathbb{R}^{n}$. For $\phi \in \mathcal{C}, \phi_{[-\Delta, 0)}$ is the function in $\mathcal{Q}$ defined, for $\tau \in[-\Delta, 0)$, as $\phi_{[-\Delta, 0)}(\tau)=\phi(\tau)$. For a positive real $p$, for $\phi \in \mathcal{C}, \mathcal{C}_{p}(\phi)=\left\{\psi \in \mathcal{C}:\|\psi-\phi\|_{\infty} \leq p\right\}$. The symbol $\mathcal{C}_{p}$ denotes $\mathcal{C}_{p}(0)$. For a continuous function $x:[-\Delta, c) \rightarrow \mathbb{R}^{n}$, with $0<c \leq+\infty$, for any real $t \in[0, c), x_{t}$ is the function in $\mathcal{C}$ defined as $x_{t}(\tau)=$ $x(t+\tau), \tau \in[-\Delta, 0]$. Let us here recall that a continuous function $\gamma: \mathbb{R}^{+} \rightarrow \mathbb{R}^{+}$is: of class $\mathcal{P}_{0}$ if $\gamma(0)=0$; of class $\mathcal{P}$ if it is of class $\mathcal{P}_{0}$ and $\gamma(s)>0, s>0$; of class $\mathcal{K}$ if it is of class $\mathcal{P}$ and strictly increasing; of class $\mathcal{K}_{\infty}$ if it is of class $\mathcal{K}$ and unbounded. The symbol $I_{d}$ denotes the identity function in $\mathbb{R}^{+}$. Throughout the paper, ISS stands for Input-to-State Stability and CLKF stands for control Lyapunov-Krasovskii functional.

\section{GLUCOSE-INSULIN MODEL}

Define $G(t),[\mathrm{mM}]$, and $I(t),[\mathrm{pM}]$, the plasma glucose and insulin concentrations. The nonlinear DDE model, [23],
[26], exploited to design the closed-loop control law is

$$
\begin{aligned}
& \dot{G}(t)=-K_{x g i} G(t) I(t)+\frac{T_{g h}}{V_{G}}, \\
& \dot{I}(t)=-K_{x i} I(t)+\frac{T_{i G \max }}{V_{I}} \varphi\left(G\left(t-\tau_{g}\right)\right)+\frac{v(t)}{V_{I}}, \\
& G(\tau)=G_{0}(\tau) \quad I(\tau)=I_{0}(\tau) \quad \tau \in\left[-\tau_{g}, 0\right]
\end{aligned}
$$

where $K_{x g i},\left[\min ^{-1} \mathrm{pM}^{-1}\right]$, is the insulin-dependent glucose uptake rate per pM of plasma insulin concentration, $T_{g h}$, $\left[\mathrm{min}^{-1}(\mathrm{mmol} / \mathrm{kgBW})\right]$, is the net balance between hepatic glucose output and insulin-independent zero-order glucose tissue uptake, $V_{G}$ and $V_{I}$, [L/KgBW], are the distribution volumes for glucose and insulin, $K_{x i}$, $\left[\mathrm{min}^{-1}\right]$, is the clearance rate constant for insulin, $T_{i G \max },\left[\mathrm{min}^{-1}(\mathrm{pmol} / \mathrm{kgBW})\right]$, is the maximal rate of second-phase insulin release, $\varphi(\cdot)$ models the Insulin Delivery Rate as the following sigmoidal function: $\varphi\left(G\left(t-\tau_{g}\right)\right)=\frac{\left(\frac{G\left(t-\tau_{g}\right)}{G^{*}}\right)^{\gamma}}{1+\left(\frac{G\left(t-\tau_{g}\right)}{G^{*}}\right)^{\gamma}}$, with $\gamma$ the sigmoidal function parameter related to the steepness of $\varphi$, $G^{*},[\mathrm{mM}]$, the glycemia at which the insulin release is the half of its maximal rate and $\tau_{g}$, [min], the apparent delay with which the pancreas varies secondary insulin release in response to varying plasma glucose concentrations. The pair $\left(G_{0}(\tau), I_{0}(\tau)\right)$ is the initial condition of the model, corresponding to the plasma glucose/insulin concentrations before the control input is applied. They can be taken equal to the constant basal levels $\left(G_{b}, I_{b}\right)$. No glucose disturbances are here considered, i.e. the patients are supposed to be at rest, away from meals immediately before and during the control therapy. Instead, disturbances in the insulin kinetics will be accounted for in the next section. $v(t),[(\mathrm{pmol} / \mathrm{kgBW}) / \mathrm{min}]$, is the exogenous intra-venous insulin delivery rate, i.e., the control input. Let $G_{r e f}$ be the desired glucose reference, the one to be tracked by the control law. The choice of a desired glucose level $G_{r e f}$ leads to the definition of the insulin and input references, $I_{\text {ref }}$ and $v_{\text {ref }}$, respectively

$$
I_{\text {ref }}=\frac{T_{g h}}{V_{G} G_{r e f} K_{x g i}}, v_{r e f}=V_{I} I_{r e f} K_{x i}-T_{i G \max } \varphi\left(G_{r e f}\right)
$$

and refers to the steady state solution achieved by fixing $v(t)=v_{\text {ref }}$. They are exploited to rewrite system (1) with respect to the displacement $x(t)=\left[\begin{array}{l}x_{1}(t) \\ x_{2}(t)\end{array}\right]=$ $\left[\begin{array}{c}G(t)-G_{r e f} \\ I(t)-I_{r e f}\end{array}\right]$, with the new control input $u(t)=v(t)-$ $v_{r e f}$. By letting $x_{t}=\left[\begin{array}{ll}x_{1, t} & x_{2, t}\end{array}\right]^{T}=\left[\begin{array}{ll}G_{t}-G_{r e f} & I_{t}-I_{r e f}\end{array}\right]^{T}$ :

$$
\begin{aligned}
& \dot{x}(t)=f\left(x_{t}\right)+g\left(x_{t}\right) u(t)=\left[\begin{array}{l}
f_{1}\left(x_{t}\right) \\
f_{2}\left(x_{t}\right)
\end{array}\right]+\left[\begin{array}{c}
0 \\
\frac{1}{V_{I}}
\end{array}\right] u(t), \\
& x(\tau)=x_{0}(\tau)=\left[\begin{array}{c}
G_{0}(\tau) \\
I_{0}(\tau)
\end{array}\right]-\left[\begin{array}{l}
G_{\text {ref }} \\
I_{\text {ref }}
\end{array}\right], \quad \tau \in\left[-\tau_{g}, 0\right],
\end{aligned}
$$

where the constant map $g: \mathcal{C} \rightarrow \mathbb{R}^{2}$, the maps $f_{i}: \mathcal{C} \rightarrow \mathbb{R}$, $i=1,2$, and, consequently, the map $f: \mathcal{C} \rightarrow \mathbb{R}^{2}$, are defined, by using the right-hand side of (1), as follows

$$
\begin{aligned}
& f_{1}\left(x_{t}\right)=-K_{x g i}\left(x_{1}(t)+G_{r e f}\right)\left(x_{2}(t)+I_{r e f}\right)+\frac{T_{g h}}{V_{G}}, \\
& f_{2}\left(x_{t}\right)=-K_{x i}\left(x_{2}(t)+I_{r e f}\right)+\frac{T_{i G \max }}{V_{I}} \varphi\left(x_{1}\left(t-\tau_{g}\right)\right.
\end{aligned}
$$




$$
\left.+G_{r e f}\right)+\frac{v_{r e f}}{V_{I}}, \quad g\left(x_{t}\right)=\left[\begin{array}{ll}
0 & \frac{1}{V_{I}}
\end{array}\right]^{T} .
$$

The equality $f(0)=0$ holds, according to (2).

\section{DESIGN OF SAMPLED-DATA GLOBAL CONTROLLERS FOR GLUCOSE-INSULIN SYSTEM}

In the following, thanks to the theory on the stabilization in the sample-and-hold sense (see [4], [5]), as applied to timedelay systems (see [28] and [29]), we provide a global nonlinear sampled-data state feedback, aiming at reducing a high basal plasma glucose concentration to a reference glucose value (i.e., no a-priori limitations on the state variables are required). Sontag's universal formula has been used in order to find the state feedback (see [17], [30], [35] and [36]). In [17], theoretical results are shown concerning Sontag's universal formula and event-based paradigm using continuoustime outputs. Instead here, Sontag's universal formula is used in order to design a global nonlinear state feedback by the use of discrete-time outputs. Furthermore, here we take into account observation errors in the measurements of glucose and insulin concentrations, and disturbances $d(t)$, affecting the insulin delivery rate and the mechanism actuating the insulin pump. In order to attenuate the effects of such disturbances and errors, we make use of the recent results about the ISS redesign methods in the framework of stabilization in the sample-and-hold sense theory (see [7], [8], [32], and, for the continuous-time case, see [33], [36]).

Taking into account (3), (4), and according to Sontag's universal formula [33], the state-feedback is here set as $u(t)=k\left(x_{t}\right)$, where $k: \mathcal{C} \rightarrow \mathbb{R}$ is defined, for $\phi \in \mathcal{C}$, as

$$
k(\phi)= \begin{cases}-\frac{\tilde{a}(\phi)+\sqrt{\tilde{a}^{2}(\phi)+\tilde{b}^{4}(\phi)}}{\tilde{b}(\phi)}, & \tilde{b}(\phi) \neq 0, \\ 0, & \tilde{b}(\phi)=0 .\end{cases}
$$

with $\tilde{a}: \mathcal{C} \rightarrow \mathbb{R}$ and $\tilde{b}: \mathcal{C} \rightarrow \mathbb{R}$ the maps defined, for $\phi=\left[\begin{array}{l}\phi_{1} \\ \phi_{2}\end{array}\right] \in \mathcal{C}$, as

$$
\begin{aligned}
& \tilde{a}(\phi)=2 \lambda_{1}(1+\eta) \phi^{T}(0) f(\phi)+\left(\eta \mu \lambda_{1}+\lambda_{2}\right)\left(\phi_{1}^{2}(0)\right. \\
& \left.\quad+\phi_{2}^{2}(0)\right)-\lambda_{2}\left(\phi_{1}^{2}\left(-\tau_{g}\right)+\phi_{2}^{2}\left(-\tau_{g}\right)\right) \\
& \tilde{b}(\phi)=2 \lambda_{1}(1+\eta) \phi_{2}(0) \frac{1}{V_{I}},
\end{aligned}
$$

with $\eta, \mu, \lambda_{1}$ and $\lambda_{2}$, positive reals, control parameters.

Before to state how to tune these parameters, we recall the notion of partition of $[0,+\infty)$, that we use in the theory of sampled-data systems (see [5] and [28]).

Definition 1: A partition $\pi=\left\{t_{i}, i=0,1, \ldots\right\}$ of $[0,+\infty)$ is a countable, strictly increasing sequence $t_{i}$, with $t_{0}=0$, such that $t_{i} \rightarrow+\infty$ as $i \rightarrow+\infty$. The diameter of $\pi$, denoted $\operatorname{diam}(\pi)$, is defined as $\sup _{i \geq 0} t_{i+1}-t_{i}$. The dwell time of $\pi$, denoted dwell $(\pi)$, is defined as $\inf _{i \geq 0} t_{i+1}-t_{i}$. For any positive real $a \in(0,1], b>0, \pi_{a, b}$ is any partition $\pi$ with $a b \leq \operatorname{dwell}(\pi) \leq \operatorname{diam}(\pi) \leq b$.

We introduce the following standard assumptions (see Assumption 1 in [28] and Assumption 3 in [7]), which will be exploited to prove the results of the paper (stated in Theorem 1 and 2). Theorem 1 is based on the theory of stabilization in the sample-and-hold sense for time-delay systems (see [28], [29]).

Assumption 1: The initial condition of system (3) $x_{0}$ is in $W^{1, \infty}$, and $e s s \sup _{\theta \in\left[-\tau_{g}, 0\right]}\left|\frac{d x_{0}(\theta)}{d \theta}\right| \leq q(q$ arbitrarily given positive real).

Assumption 2: The positive reals $\eta, \mu, \lambda_{1}, \lambda_{2}$ and the corresponding maps $\tilde{a}, \tilde{b}, k$, defined in (5), (6), are such that:

$$
\mu \lambda_{1}>\lambda_{2} ; \quad \tilde{b}(\phi)=0 \Rightarrow \tilde{a}(\phi) \leq 0, \quad \forall \phi=\left[\begin{array}{l}
\phi_{1} \\
\phi_{2}
\end{array}\right] \in \mathcal{C} .
$$

Theorem 1: Let Assumptions 1 and 2 hold. Then, for any positive reals $r, R, 0<r<R, a \in(0,1]$, there exist positive reals $\delta, T$ and $E$ such that, for any partition $\pi_{a, \delta}=$ $\left\{t_{i}, i=0,1, \ldots\right\}$, for any initial state $x_{0} \in \mathcal{C}_{R}$, the solution of system (3), corresponding to $x_{0}$ and to the sampleddata feedback control law $u(t)=k\left(x_{t_{j}}\right), t_{j} \leq t<t_{j+1}$, $j=0,1, \ldots$, exists for all $t \geq 0$ and, furthermore, satisfies the following inequalities

$$
\left|\begin{array}{l}
x_{1}(t) \\
x_{2}(t)
\end{array}\right| \leq E, \quad \forall t \geq 0, \quad\left|\begin{array}{l}
x_{1}(t) \\
x_{2}(t)
\end{array}\right| \leq r, \quad \forall t \geq T .
$$

Proof: The proof is reported in Subsection IV-A.

The following theorem shows the robustness of the proposed control in spite of the aforementioned uncertainties.

Theorem 2: Let Assumptions 1 and 2 hold. Let $S: \mathcal{C} \rightarrow \mathbb{R}$ be the map defined, for $\phi \in \mathcal{C}$, as

$$
S(\phi)=-2 \lambda_{1} \omega \phi_{2}(0) \frac{1}{V_{I}},
$$

where $\omega$ is a further control tuning parameter.

Then, for any positive reals $\bar{d}, \bar{e}, r, R, 0<r<R, a \in(0,1]$, there exist a positive real $E>R$ and a positive real $\underline{\omega}$ such that, for any $\omega \geq \underline{\omega}$, there exist positive reals $\delta$ and $T$ such that: for any partition $\pi_{a, \delta}=\left\{t_{i}, i=0,1, \ldots\right\}$, for any initial state $x_{0} \in \mathcal{C}_{R}$, for any sequence $d: \mathbb{N} \rightarrow \mathbb{R}$ (actuation disturbance) satisfying $|d(j)| \leq \bar{d}, j=0,1, \ldots$, for any sequence $e: \mathbb{N} \rightarrow \mathcal{C}$ (observation error), satisfying $\left\|e_{j}\right\|_{\infty} \leq \bar{e}, j=0,1, \ldots$ and $\frac{2 \lambda_{1}}{V_{I}}\left|\left[\begin{array}{ll}0 & 1\end{array}\right] e(0)\right| \leq \frac{\bar{e}}{\omega}$, the solution of the system (3), corresponding to $x_{0}$ and to the input $u(t)=k\left(x_{t_{j}}+e_{j}\right)+S\left(x_{t_{j}}+e_{j}\right)+d(j), t_{j} \leq t<$ $t_{j+1}$, exists $\forall t \geq 0$ and, furthermore, satisfies:

$$
\left|\begin{array}{l}
x_{1}(t) \\
x_{2}(t)
\end{array}\right| \leq E, \quad \forall t \geq 0, \quad\left|\begin{array}{l}
x_{1}(t) \\
x_{2}(t)
\end{array}\right| \leq r, \quad \forall t \geq T .
$$

Proof: The proof is reported in Subsection IV-B.

Remark 1: We stress that the actuation disturbances and the observation errors are unknown. It is only necessary to know the related (arbitrary as long as finite) upper bounds. Moreover, it is required that the observation errors do not affect, or affect marginally the new added control term $S$ (see (9)). The case of suitably small observation errors is always covered. The reader can refer to [32] for a related discussion in the case of finite dimensional nonlinear systems. In the developed theory, we do not take into account input saturation constraints. The control input $v(t)$ cannot be negative, since the insulin pump cannot draw insulin from the patient. We take saturation constraints into account in the performed simulations. 


\section{PROOFS OF THEOREMS 1,2}

\section{A. Proof of Theorem 1}

In order to prove Theorem 1, thanks to the results proved in [28], we have to check that Assumption 1 in [28] holds for the glucose-insulin system (3), and the global state feedback $k$ defined in (5). Indeed, if Assumption 1 in [28] holds, then the global state feedback $k$ provided in (5) is a steepest descent feedback (see Definition 4.2 in [28]), induced by a suitable CLKF (see Definition 4.1 in [28]), for the system (3), and thus Theorem 1 follows from Theorem 5.3 in [28]. According to Assumption 1 in [28], we have to prove that there exist a CLKF $V$, according to Definition 4.1 in [28], such that: (1) the feedback $k$, as defined in (5), is a related steepest descent feedback according to Definition 4.2 in [28]; (2) the map $(\phi, u) \rightarrow D^{+} V_{2}(\phi, u)$ is Lipschitz on bounded subsets of $\mathcal{C} \times \mathbb{R}^{m}$ (see Assumption 1 in [28]). Let $V_{1}: \mathbb{R}^{n} \rightarrow$ $\mathbb{R}^{+}$be the function defined, for $x=\left[\begin{array}{ll}x_{1} & x_{2}\end{array}\right]^{T} \in \mathbb{R}^{2}$, as

$$
V_{1}(x)=\lambda_{1}\left(x_{1}^{2}+x_{2}^{2}\right) .
$$

Let $V_{2}: \mathcal{C} \rightarrow \mathbb{R}^{+}$be the functional defined, for $\phi=$ $\left[\begin{array}{ll}\phi_{1} & \phi_{2}\end{array}\right]^{T} \in \mathcal{C}$, as $V_{2}(\phi)=\int_{-\tau_{g}}^{0} \lambda_{2}\left(\phi_{1}^{2}(\tau)+\phi_{2}^{2}(\tau)\right) d \tau$. Let $V: \mathcal{C} \rightarrow \mathbb{R}^{+}$be the functional defined, for $\phi \in \mathcal{C}$, as $V(\phi)=V_{1}(\phi(0))+V_{2}(\phi)$. Let $\beta_{i}, i=1,2$, be the functions of class $\mathcal{K}_{\infty}$ defined, for $s \in \mathbb{R}^{+}$, as

$$
\beta_{1}(s)=\beta_{2}(s)=\lambda_{1} s^{2} .
$$

Then, the functional $V$ is smoothly separable, according to Definition 3.1 in [28] (with the functions $\beta_{i}, i=1,2$, defined in (12)). The point (2) is clearly satisfied. Let $\gamma_{i}, i=1,2$, be the functions of class $\mathcal{K}_{\infty}$ defined, for $s \in \mathbb{R}^{+}$, as

$$
\gamma_{1}(s)=\lambda_{1} s^{2}, \quad \gamma_{2}(s)=\left(\lambda_{1}+\tau_{g} \lambda_{2}\right) s^{2} .
$$

Then, the functional $V$ satisfies the inequalities (i) of Definition 4.1 in [28] with the functions $\gamma_{i}, i=1,2$, defined in (13). It remains to prove that inequalities (ii) and (4.1), stated in Definitions 4.1 and 4.2 in [28], hold (i.e. point (1) holds). We will prove first the inequality (4.1) of Definition 4.2 in [28]. Let us choose $p=I_{d}, \nu=\bar{p}=1, \bar{\alpha}=0$. We have two cases: a) $D^{+} V_{1}(\phi, k(\phi))+\mu V_{1}(\phi(0)) \leq 0$; b) $D^{+} V_{1}(\phi, k(\phi))+\mu V_{1}(\phi(0))>0$. If the case (a) holds, then, taking into account (3), (4), (5), for all $\phi \in \mathcal{C}$, the following inequality holds

$$
\begin{aligned}
& 2 \lambda_{1}\left(\phi_{1}(0) f_{1}(\phi)+\phi_{2}(0) f_{2}(\phi)+\frac{\phi_{2}(0)}{V_{I}} k(\phi)\right) \\
& +\mu \lambda_{1}\left(\phi_{1}^{2}(0)+\phi_{2}^{2}(0)\right) \leq 0 .
\end{aligned}
$$

Now, taking into account (3), (4), (5), (7), (14), the following equalities/inequalities hold

$$
\begin{aligned}
& D^{+} V(\phi, k(\phi))= \\
& 2 \lambda_{1}\left(\phi_{1}(0) f_{1}(\phi)+\phi_{2}(0) f_{2}(\phi)+\frac{\phi_{2}(0)}{V_{I}} k(\phi)\right) \\
& +\lambda_{2}\left(\phi_{1}^{2}(0)+\phi_{2}^{2}(0)\right)-\lambda_{2}\left(\phi_{1}^{2}\left(-\tau_{g}\right)+\phi_{2}^{2}\left(-\tau_{g}\right)\right)= \\
& 2 \lambda_{1}\left(\phi_{1}(0) f_{1}(\phi)+\phi_{2}(0) f_{2}(\phi)+\frac{\phi_{2}(0)}{V_{I}} k(\phi)\right) \\
& +\lambda_{2}\left(\phi_{1}^{2}(0)+\phi_{2}^{2}(0)\right)-\lambda_{2}\left(\phi_{1}^{2}\left(-\tau_{g}\right)+\phi_{2}^{2}\left(-\tau_{g}\right)\right) \\
& +\mu \lambda_{1}\left(\phi_{1}^{2}(0)+\phi_{2}^{2}(0)\right)-\mu \lambda_{1}\left(\phi_{1}^{2}(0)+\phi_{2}^{2}(0)\right) \\
& \leq\left(\lambda_{2}-\mu \lambda_{1}\right)\left(\phi_{1}^{2}(0)+\phi_{2}^{2}(0)\right) \leq 0 .
\end{aligned}
$$

Therefore, inequality (4.1) of Definition 4.2 in [28] holds in the case (a). As far as the case (b) is concerned, taking into account (3), (4), (5), (7), the following equalities/inequality hold:

$$
\begin{aligned}
& D^{+} V(\phi, k(\phi))+\eta D^{+} V_{1}(\phi, k(\phi))+\eta \mu V_{1}(\phi(0))= \\
& 2 \lambda_{1}\left(\phi_{1}(0) f_{1}(\phi)+\phi_{2}(0) f_{2}(\phi)+\frac{\phi_{2}(0)}{V_{I}} k(\phi)\right) \\
& +\lambda_{2}\left(\phi_{1}^{2}(0)+\phi_{2}^{2}(0)\right)-\lambda_{2}\left(\phi_{1}^{2}\left(-\tau_{g}\right)+\phi_{2}^{2}\left(-\tau_{g}\right)\right) \\
& +2 \eta \lambda_{1}\left(\phi_{1}(0) f_{1}(\phi)+\phi_{2}(0) f_{2}(\phi)+\frac{\phi_{2}(0)}{V_{I}} k(\phi)\right) \\
& +\eta \mu \lambda_{1}\left(\phi_{1}^{2}(0)+\phi_{2}^{2}(0)\right)= \\
& 2 \lambda_{1}(1+\eta)\left(\phi_{1}(0) f_{1}(\phi)+\phi_{2}(0) f_{2}(\phi)+\frac{\phi_{2}(0)}{V_{I}} k(\phi)\right) \\
& +\left(\eta \mu \lambda_{1}+\lambda_{2}\right)\left(\phi_{1}^{2}(0)+\phi_{2}^{2}(0)\right) \\
& -\lambda_{2}\left(\phi_{1}^{2}\left(-\tau_{g}\right)+\phi_{2}^{2}\left(-\tau_{g}\right)\right)=\tilde{a}(\phi)+\tilde{b}(\phi) k(\phi) \\
& =-\sqrt{\tilde{a}^{2}(\phi)+\tilde{b}^{4}(\phi)} \leq 0 .
\end{aligned}
$$

Therefore, inequality (4.1) of Definition 4.2 in [28] is also satisfied in the case (b). From inequalities (15) and (16) it follows that, if $\phi(0) \neq 0$, then $D^{+} V(\phi, k(\phi))<0$. Therefore, the inequality (ii) of Definition 4.1 in [28] holds. The point (1) is proved. The proof of Theorem 1 is complete.

\section{B. Proof of Theorem 2}

In order to prove Theorem 2, thanks to the results proved in [7], we have to check that Assumption 2 in [7] (see also Assumption 3 in [8]) holds for the glucose-insulin system (3), and the global state feedback $k$ defined in (5). According to Assumption 2 in [7], let $V_{1}: \mathbb{R}^{n} \rightarrow \mathbb{R}^{+}$ be the function defined in (11). Let $V_{2}: \mathcal{Q} \rightarrow \mathbb{R}^{+}$ be the functional defined, for $\phi=\left[\begin{array}{ll}\phi_{1} & \phi_{2}\end{array}\right]^{T} \in \mathcal{Q}$, as $V_{2}(\phi)=\int_{-\tau_{g}}^{0} \lambda_{2}\left(\phi_{1}^{2}(\tau)+\phi_{2}^{2}(\tau)\right) d \tau$. Let $\widetilde{V}_{2}: \mathcal{C} \rightarrow \mathbb{R}^{+}$ be the functional defined, for $\phi=\left[\begin{array}{ll}\phi_{1} & \phi_{2}\end{array}\right]^{T} \in \mathcal{C}$, as $\widetilde{V}_{2}(\phi)=V_{2}\left(\phi_{\left[-\tau_{g}, 0\right)}\right)=\int_{-\tau_{g}}^{0} \lambda_{2}\left(\phi_{1}^{2}(\tau)+\phi_{2}^{2}(\tau)\right) d \tau$. Let $V: \mathcal{C} \rightarrow \mathbb{R}^{+}$be the functional defined, for $\phi \in \mathcal{C}$, as $V(\phi)=V_{1}(\phi(0))+\widetilde{V}_{2}(\phi)$. Let $\beta_{i}, i=1,2$, be the functions of class $\mathcal{K}_{\infty}$ defined in (12). Let $\gamma_{i}, i=1,2$, be the functions of class $\mathcal{K}_{\infty}$ defined in (13). The functional $V$ is smoothly separable, according to Definition 3.1 in [28] (with the functions $\beta_{i}, i=1,2$, defined in (12), for $V_{1}$ ). Therefore the points (1) and (2) of Assumption 2 in [7] hold. The point (3) of Assumption 2 in [7] holds too, taking into account $V_{2}$, and the definition of invariantly differentiable functionals (see [13], see also Definition 1 in [33]). As far as the point (4) of Assumption 2 in [7] is concerned, it holds with the functions $\gamma_{i}, i=1,2$ defined in (13). As far as point (5) is concerned, let us choose $p, \nu, \bar{p}, \bar{\alpha}$ as in the proof of Theorem 1. Then, the same steps used in the proof of Theorem 1 (from (14) to (16)) can be used here, for proving the point (5) of Assumption 2 in [7] with the functional $V_{1}$ defined in (11), and the functional $V$ here defined. The proof of Theorem 2 is complete.

\section{APPLICATION TO A T2DM PATIENT}

In this section, an application of Theorems 1 and 2 in the case of a T2DM patient is proposed, with the patient modeled by the DDE system (1) with model parameters taken 
from [25] and reported in Table I. Taking into account (2),

TABLE I

Parameters

\begin{tabular}{|c|c|c|c|}
\hline \multicolumn{4}{|c|}{ Parameters } \\
\hline$G_{b}=$ & $I_{b}=48.95$ & $\overline{T_{i G \max }}=0.2$ & \\
\hline $\begin{array}{r}V_{G}=0.187 \\
\quad V_{I}=0.25\end{array}$ & $\begin{array}{c}K_{x i}=1.211 \cdot 10^{-2} \\
K_{x g i}=3.11 \cdot 10^{-5}\end{array}$ & $\begin{array}{l}T_{g h}=0.003 \\
\gamma=3.205\end{array}$ & $\begin{array}{l}\tau_{g}=24 \\
G^{*}=9\end{array}$ \\
\hline
\end{tabular}

we obtain $I_{\text {ref }}=109.7543$ and $v_{\text {ref }}=0.3055$. Choosing $\eta=1.5, \mu=0.5 \cdot 10^{-2}, \lambda_{1}=3 \cdot 10^{-3}$ and $\lambda_{2}=10^{-5}$, Assumption 2 holds. Indeed, taking into account the first condition in (7), it holds true since $\mu \lambda_{1}=1.5 \cdot 10^{-5}>\lambda_{2}=$ $10^{-5}$. Regards the second condition in (7) (see also (6)), we have: $\tilde{b}(\phi)=15 \cdot 10^{-3} \frac{\phi_{2}(0)}{V_{I}}=0 \quad \Longrightarrow \quad \phi_{2}(0)=0$, that means

$$
\begin{aligned}
& \tilde{a}(\phi)=15 \cdot 10^{-3} \phi_{1}(0) f_{1}(\phi)+3.25 \cdot 10^{-5} \phi_{1}^{2}(0) \\
& -10^{-5}\left(\phi_{1}^{2}\left(-\tau_{g}\right)+\phi_{2}^{2}\left(-\tau_{g}\right)\right) \leq 15 \cdot 10^{-3} \phi_{1}(0) \\
& \cdot\left(-K_{x g i} I_{r e f}\left(\phi_{1}(0)+G_{r e f}\right)+\frac{T_{g h}}{V_{G}}\right)+3.25 \cdot 10^{-5} \phi_{1}^{2}(0) \\
& \leq-15 \cdot 10^{-3} \phi_{1}^{2}(0)\left(K_{x g i} I_{r e f}-\frac{3.25 \cdot 10^{-5}}{15 \cdot 10^{-3}}\right) \\
& +15 \cdot 10^{-3} \phi_{1}(0)\left(-K_{x g i} G_{r e f} I_{r e f}+\frac{T_{g h}}{V_{G}}\right) \leq 0,
\end{aligned}
$$

according to the chosen set of model parameters reported in Table I. We choose the initial state, according to Table I and (2), for $\tau \in\left[-\tau_{g}, 0\right]$ as $x_{0}(\tau)=$ $\left[\begin{array}{ll}G_{b}-G_{r e f} & I_{b}-I_{r e f}\end{array}\right]^{T}=\left[\begin{array}{ll}5.67 & -60.8043\end{array}\right]^{T}$. The initial state satisfies Assumption 1. Thus, all the assumptions, needed to apply the theoretical results stated in Theorems 1 and 2, are satisfied for the patient in exam. The proposed simulation shows the good performances of the global sampled-data state feedback $k$, provided in (5) (see also (3), (4)), for a sampling period $\delta=15 \mathrm{~min}$ (Fig. 1): plasma glycemia is constrained below $7[\mathrm{mM}]$ within the first 3 hours of the treatment, according to a smooth trajectory that avoids dangerous oscillations. Moreover, the saturation effect is evident in Fig. 1. This is due to the fact that the high insulin delivery rate computed at $\mathrm{t}=0$ is applied over a relatively long sampling period. Nevertheless, the behavior of the closed loop glucose-insulin system is acceptable, since we are far from dangerous hypoglycemias $(G(t)<3.3[\mathrm{mM}])$. Thanks to Theorem 2, the term to be added to the feedback $k$ (see (5)), in order to ensure stabilization, despite actuation disturbances and observation errors, is given by (9). In performed simulations the actuator disturbance $d(j)=0.15 \sin \left(0.05 t_{j}\right), j=0,1, \ldots$, is chosen. Disturbance amplitude is equal to $49 \%$ of $v_{r e f}$. As far as the observation error is concerned, we considered $e_{j}=$ $\left[\begin{array}{ll}e_{1, j} & e_{2, j}\end{array}\right]^{T} \in \mathcal{C}$, with (as necessary terms for simulations) $e_{1, j}(0)=e_{1, j}\left(-\tau_{g}\right)=\tilde{e}_{1}$ and $e_{2, j}(0)=e_{2, j}\left(-\tau_{g}\right)=\tilde{e}_{2}$, with $\tilde{e}_{1}$ and $\tilde{e}_{2}$ taken from the interval $[-0.1,0.1]$ and $[-0.15,0.15]$, respectively, by emulation of the uniform probability density functions. The proposed simulation shows the robustness of the global sampled-data state feedback. In Fig. 2 the glucose and insulin levels, in both cases of robustified controller (continuous line) and non robustified controller (dashed line) are reported, respectively. Moreover,
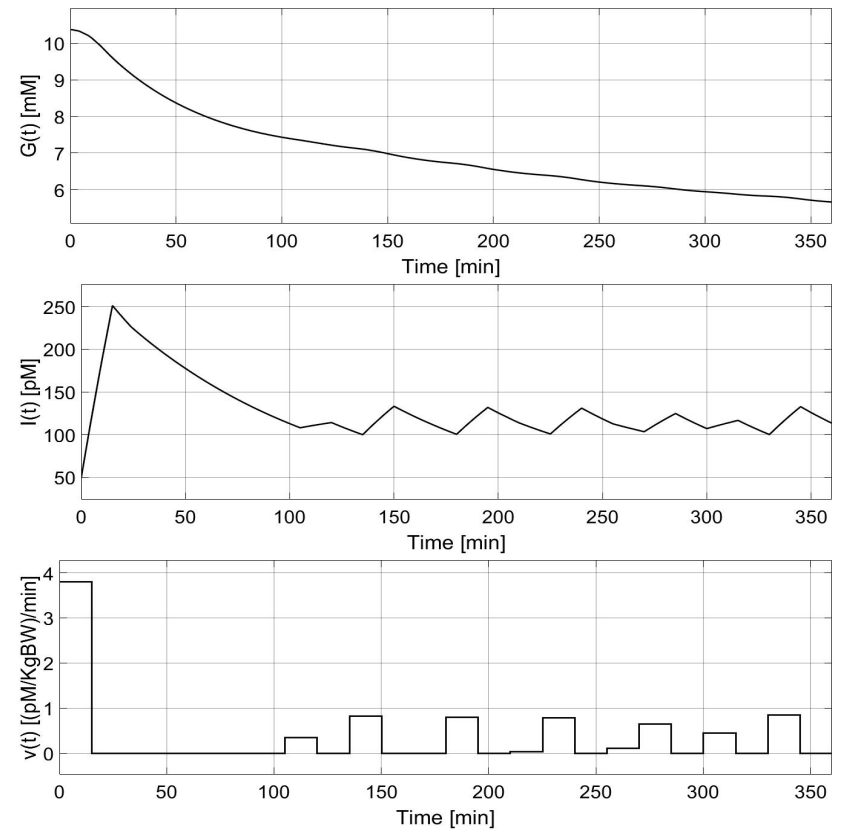

Fig. 1. Simulation on a T2DM patient with $\delta=15 \mathrm{~min}$.

the control signal in the case of robustified controller, with a sampling period $\delta=4 \min$ is plotted. We adjusted the tuning parameter by some experiments and set $\omega=1.5$. In the simulation, we notice how the new robustified controller can cope with the effect of actuation disturbances and observation errors, in both the transient and the steady-state response. Moreover, by reducing the sampling period, the controller rarely violates saturation constraints, and the disturbance attenuation becomes more evident. According to not reported simulations we stress that the controller would have worked as well also if the variability of parameters and additional events such as the evolution of glucose during a physical activity are considered.

\section{CONCLUSION}

In this paper, the recent results about stabilization in the sample-and-hold sense, for time-delay systems, have been used in order to cope with the problem related to the tracking of a desired plasma glucose concentration by means of intravenous insulin administration for T2DM patients. Sontag's universal formula has been used in order to obtain a nonlinear global state feedback. Such feedback has been used as a stabilizer in the sample-and-hold sense for the glucoseinsulin system. Performed simulations have validated the proposed closed-loop glucose control strategy. Furthermore, the ISS redesign method has been used in order to cope with the effects of observation errors in the measurements of glucose and insulin levels, and disturbances, affecting the insulin delivery rate and the mechanism actuating the insulin pump. The simulations show the robustness of the controller with respect to actuation disturbances and observation errors. A satisfactory behavior of the glucose evolution is obtained. 

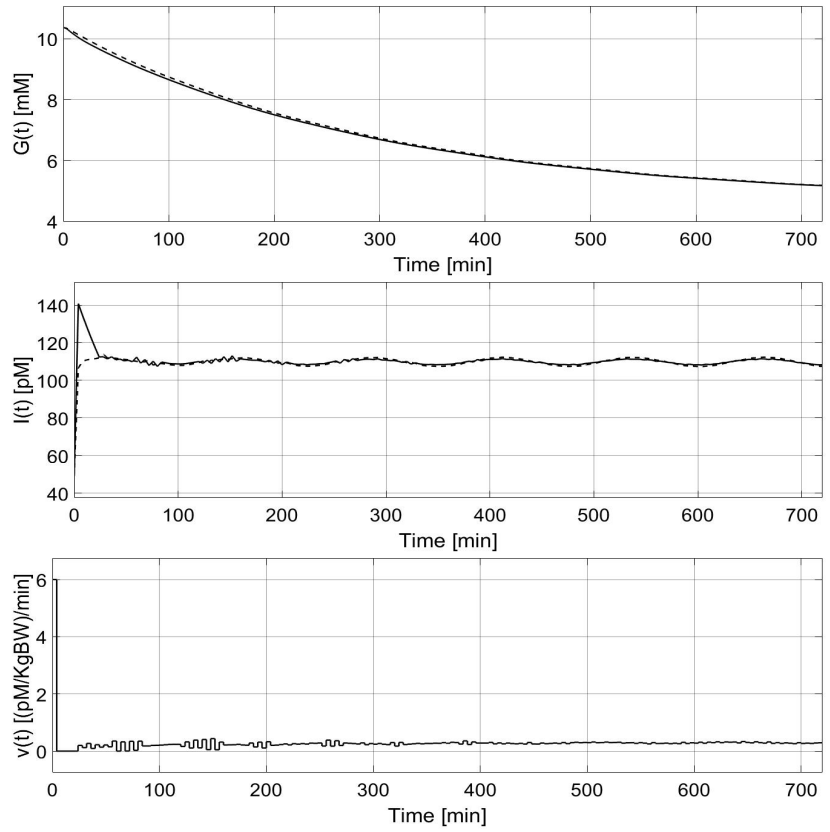

Fig. 2. Simulation on a T2DM patient with $\delta=4 \mathrm{~min}$, in the case of robustified (continuous line) and non-robustified (dashed line) controller.

\section{REFERENCES}

[1] International-Diabetes-Federation, IDF Diabetes Atlas, Brussels, Belgium, 2013.

[2] D. Carnevale, A. R. Teel and D. Nesic , "A Lyapunov proof of an improved maximum allowable transfer interval for networked control systems", IEEE Trans. Automat. Control, vol. 52, 2007, pp 892-897.

[3] F. Chee, A. V. Savkin, T.L. Fernando, S. Nahavandi, Optimal H-infinity insulin injection control for blood glucose regulation in diabetic patients, IEEE Trans. on Biomedical Engineering, vol. 52, 2005, pp 1625-1631.

[4] F. H. Clarke, "Discontinuous feedback and nonlinear systems", Plenary Lecture at IFAC Conference on Nonlinear Control Systems, 2010.

[5] F. H. Clarke, Y. S. Ledyaev, E. D. Sontag, A. I. Subbotin, ”Asymptotic controllability implies feedback stabilization", IEEE Trans. Automat. Control, vol. 42, 1997, pp 1394-1407.

[6] C. Cobelli, E. Renard, B. Kovatchev, Artificial pancreas: past, present, future, Diabetes, vol. 60, 2011, pp 2672-2682.

[7] M. Di Ferdinando, P. Pepe, "Robust Sample-and-Hold Stabilization for Nonlinear Retarded Systems", IFAC-PapersOnLine , vol. 49, 2016, pp 53-58.

[8] M. Di Ferdinando, P. Pepe, "Robustification of Sample-and-Hold Stabilizers for Control-Affine Time-Delay Systems", Automatica, vol. 83, 2017, pp 141-154.

[9] F. J. Doyle III, L.M. Huyett, J.B. Lee, H.C. Zisser, E. Dassau, Closed-Loop Artificial Pancreas Systems: Engineering the Algorithms, Diabetes Care, vol. 37, 2014, pp 1191-1197.

[10] P. Dua, F.J. Doyle III, E.N. Pistikopoulos, Model-based blood glucose control for Type 1 diabetes via parametric programming, IEEE Trans. Biomed. Eng., vol. 53, 2006, pp 1478-1491.

[11] E. Fridman, "Introduction to Time-Delay Systems: Analysis and Control", Birkhauser, 2014.

[12] R. Hovorka, "Closed-loop insulin delivery: From bench to clinical practice", Nature Rev. Endocrinol., vol. 7(7), 2011, pp 385-395.

[13] A. V. Kim, "On the Lyapunov's functionals method for systems with delays", Nonlin. Anal., Theory, Methods and Applic., vol. 28, 1997, pp 673-687.

[14] L. Kovàcs, P. Szalay, Z. Almàssy, L. Barkai, "Applicability Results of a Nonlinear Model-Based Robust Blood Glucose Control Algorithm", J. Diabetes Science and Technology, vol. 7(3), 2013, pp 708-716.

[15] B. Kovatchev, W.V. Tamborlane, W.T. Cefalu, C. Cobelli, "The Artificial Pancreas in 2016: A Digital Treatment Ecosystem for Diabetes", Diabetes Care, vol. 39, 2016, pp 1123-1126.
[16] A. Makroglou, J. Li, Y. Kuang, ”Mathematical models and software tools for the glucose-insulin regulatory system and diabetes: an overview", Applied Numerical Mathematics, vol. 56, 2006, pp 559573.

[17] S. Durand, N. Marchand, J. F. Guerrero Castellanos, "General Formula for Event-Based Stabilization of Nonlinear Systems with Delays in the State", Delays and Networked Control Systems, Springer, Advances in Delays and Dynamics, vol. 6, 2016, pp 59-77.

[18] D. Nesic and A. R. Teel, "Sampled-data control of nonlinear systems: an overview of recent results", Perspectives on Robust Control, 2001, pp 221-239.

[19] D. Nesic and A. R. Teel, "A framework for stabilization of nonlinear sampled-data systems based on their approximate discrete-time models", IEEE Trans. Automat. Control, vol. 49, 2004, pp 1103-1122.

[20] D. Nesic, A. R. Teel and D. Carnevale, "Explicit Computation of the Sampling Period in Emulation of Controllers for Nonlinear SampledData Systems", IEEE Trans. Automat. Control, vol. 54, 2009, pp 619624

[21] P. Palumbo, S. Ditlevsen, A. Bertuzzi, A. De Gaetano, Mathematical modeling of the glucoseinsulin system: A review, Math. Biosci., vol. 244, 2013, pp 69-81.

[22] P. Palumbo, G. Pizzichelli, S. Panunzi, P. Pepe, A. De Gaetano, Modelbased control of plasma glycemia: Tests on populations of virtual patients, Math. Biosciences, vol. 257, 2014, pp 2-10.

[23] P. Palumbo, S. Panunzi, A. De Gaetano, "Qualitative behavior of a family of delay differential models of the glucose insulin system", Discrete and Continuous Dynam. Systems - B, vol. 7, 2007, pp 399424.

[24] P. Palumbo, P. Pepe, S. Panunzi, A. De Gaetano, Time-Delay ModelBased Control of the Glucose-Insulin System, by Means of a State Observer, European Journal of Control, vol. 6, 2012, pp 591-606.

[25] P. Palumbo, P. Pepe, S. Panunzi, A. De Gaetano, "Robust closedloop control of plasma glycemia: a discrete-delay model approach", Discrete and Continuos Dynamical Systems, vol. 12, 2009, pp 455468.

[26] S. Panunzi, P. Palumbo, A. De Gaetano, ”A discrete single delay model for the intra-venous glucose tolerance test", Theoretical Biology and Medical Modelling, vol. 4, 2007.

[27] R. S. Parker, F.J. Doyle III, J.H. Ward, N.A. Peppas, Robust Hinfinity glucose control in diabetes using a physiological model, AIChE Journal, vol. 46, 2000, pp 2537-2549.

[28] P. Pepe, "Stabilization in the sample-and-hold sense of nonlinear retarded systems", SIAM J. Control Optim., vol. 52, 2014, pp 30533077.

[29] P. Pepe, "On stability preservation under sampling and approximation of feedbacks for retarded systems", SIAM J. Control Optim., vol. 54 , 2016, pp 1895-1918

[30] P. Pepe, "On Sontag's formula for the input-to-state practical stabilization of retarded control-affine systems", Syst. Control Lett., vol. 62, 2013, pp. 1018-1025.

[31] P. Pepe, P. Palumbo, S. Panunzi, A. De Gaetano, "Local SampledData Control of the Glucose-Insulin System", Proc. American Control Conference (ACC17), pp.110-115, Seattle (WA), 2017.

[32] P. Pepe, "Robustification of nonlinear stabilizers in the sample-andhold sense", Journal of The Franklin Institute, vol. 42, 2015, pp. 1394 1407.

[33] P. Pepe, H. Ito, "On saturation, discontinuities and delays, in iISS and ISS feedback control redesign", IEEE Trans. Automat. Control, vol. 57, 2012, pp. 1125-1140.

[34] E. RuizVelazquez, R. Femat, D. U. CamposDelgado, Blood glucose control for type I diabetes mellitus: A robust $\mathrm{H}$-infinity tracking problem, Control Engineering Practice,vol. 12, 2004, pp. 1179-1195.

[35] E. D. Sontag, "A "universal" construction of Artsteins theorem on nonlinear stabilization", Systems Control Lett., vol. 13, 1989, pp 117123 .

[36] E. D. Sontag, "Smooth stabilization implies coprime factorization", IEEE Trans. Automat. Control, vol. 34, 1989, pp. 435-443.

[37] G. Van den Berghe, Insulin therapy for the critically ill patient, Clinical Cornerstone, vol. 5(2), 2003, pp 56-63.

[38] K. Zarkogianni, E. Litsa, K. Mitsis, P.-Y. Wu, C.D. Kaddi, C.-W. Cheng, M.D. Wang, K.S. Nikita, A Review of Emerging Technologies for the Management of Diabetes Mellitus, IEEE Trans. Biomed. Eng. vol. 62(12), 2015, pp 2735-2749. 